\title{
Frequency of intra-abdominal organ injury is higher in patients with concomitant stab wounds to other anatomical areas
}

\author{
Igor Jeroukhimov ${ }^{1 * \dagger} \mathbb{B}$, Itay Wiser ${ }^{1 \dagger}$, Yehuda Hershkovitz ${ }^{1}, Z^{2}$ ahar Shapira', Kobi Peleg ${ }^{2}$, Ricardo Alfici ${ }^{3}$, Adi Givon², \\ Israeli Trauma Group and Boris Kessel ${ }^{4}$
}

\begin{abstract}
Background: Management of stable patients with abdominal stab wound remains controversial, particularly for those with no clear indications for explorative laparotomy. We evaluated the risk of intra-abdominal injury in stab wound victims concomitantly stabbed in other anatomical body areas.

Methods: We performed a retrospective cohort study of patients with abdominal stab wounds recorded in the Israeli National Trauma Registry from January 1st, 1997, to December 31st, 2013. Patients with an isolated abdominal stab wound were compared to those with concomitant stab wounds to other anatomical areas. Intra-abdominal organ injury was defined by imaging or surgery findings. Multivariate analysis using a logistic regression model was conducted to assess independent risk for intra-abdominal organ injury.

Results: The study included 3964 patients. After controlling for age, gender and hypotension on arrival, patients with multi-regional stab wounds had an increased risk of intra-abdominal injury (OR=1.3, Cl 95\% 1.1-1.6, $p<0.001)$. These patients also had a higher rate of injury to the solid organs than patients with an isolated abdominal stab wound.

Conclusions: Patients with multi-regional stab wounds have an increased risk of intra-abdominal injury. Worldwide accepted "clinical follow up" protocol may not be appropriate in management of patients with multi-regional stab wounds.
\end{abstract}

Keywords: Stab wound, Penetrating abdominal organ injury, Abdominal trauma

\section{Background}

Penetrating abdominal trauma continues to be a major cause of trauma admissions worldwide [1]. Non-operative management is a widely accepted approach for the stable patient with an abdominal stab wound who does not require urgent surgery [2-4].

\footnotetext{
* Correspondence: igorjer65@gmail.com

The work was performed at the National Center for Trauma and Emergency Medicine Research, Gertner Institute for Epidemiology and Health Policy Research, Tel Hashomer, Israel

${ }^{+}$Igor Jeroukhimov and Itay Wiser contributed equally to this work.

${ }^{1}$ Trauma Unit, Assaf Harofeh Medical Center, Zerifin 7030000, Israel, affiliated with the Sackler Faculty of Medicine, Tel Aviv University, Tel Aviv, Israel

Full list of author information is available at the end of the article
}

Clinical observation of stable patients with anterior abdominal stab wounds with no signs of peritonitis has been adopted by most of the trauma centers [5].

Currently, the management approach for patients with multi-regional stab wounds including the abdomen is not distinguished from those with isolated abdominal stab wounds.

We assume that the management approach to the patients with multiple abdominal stab wound may be different from those with isolated abdominal stab wound. That was intuitively based on idea that multiple stab wounds have potentially worse mechanism of injury and higher probability to penetrate peritoneal cavity and cause more damage. We hypothesized that the rate of intra-abdominal injury is higher in patients with multi-regional stab wounds compare to patients with isolated abdominal stab wounds. 
Our aim was to find out whether an association exists between intra-abdominal injury and other anatomical area involvement in stab wound injury.

\section{Methods}

\section{Study population}

We conducted a retrospective cohort study of trauma patients with abdominal stab wounds admitted to 19 Trauma Centers between the years 1997 and 2013. Data was obtained from the records of the Israeli National Trauma Registry maintained by Israel's National Center for Trauma and Emergency Medicine Research, Gertner Institute for Epidemiology and Health Policy Research. This institute records information concerning trauma patients hospitalized in 19 hospitals of which six are Level I, and 13 are Level II Trauma Centers. Data was obtained from the Israeli National Trauma Registry and included only anonymous information regarding the patients with no possibility of identification for the purposes of quality assessment and research. Ethical approval was obtained from the Helsinki Committee at Sheba Medical Center where the Israeli Trauma Registry located (Approval Number 5138-18-SMC).

The study population was divided into two groups: isolated abdominal stab wounds and abdominal stab wound with concomitant multi-regional stab wounds (defined as one or more concomitant stab wounds located in an anatomic area other than the abdomen). Knife was the only assaulting instrument. Patients with isolated thoraco-abdominal stab wound and patients with multiple abdominal stab wounds were excluded from the study.

Data collected included: age, gender, presence and location of intra-abdominal injury according to ICD-9 diagnosis, Injury Severity Score (ISS), Abbreviated Injury Score (AIS), systolic blood pressure on admission, Glasgow Comma Scale (GCS), hospital length of stay (LOS) and mortality.

Patients with admission systolic blood pressure $90 \mathrm{mmHg}$ or less were defined as hemodynamically unstable. In case of hemodynamic instability, presence of peritoneal signs, or evisceration of abdominal content all the victims were referred directly to operating room. In absence of absolute indications for surgery, the treatment algorithm included observation with serial physical examinations, local wound exploration, computed tomography and diagnostic laparoscopy in selected cases depends of particular hospital protocol.

We compared the presence of abdominal penetration and the other variables between both groups.

\section{Data analysis}

Statistical analysis was performed using the SAS statistical software (SAS, Cary, NC, version 9.2). Statistical tests included chi-square tests for categorical data and the Wilcoxon nonparametric test for continuous variables that were not normally distributed. A multivariate analysis was conducted using binary log two-tailed. Univariate and multivariate odds ratios appear for all variables (unadjusted - univariate, adjusted - multivariate).

\section{Results \\ Participants}

We found 438,765 trauma victims in the Israeli National Trauma Registry between the years 1997 to 2013. Of these, 3964 suffered from abdominal stab wounds $(0.9 \%)$. Baseline characteristics of both groups are presented in Table 1. No significant differences were observed between groups in age and gender. The multi-regional stab wounds group had a significantly higher rate of hypotension on arrival and GCS $\leq 8$ compared to the group with an isolated stab wound $(9.3 \%$ vs $5.5 \%, p<0.0001$ and $4.1 \%$ vs. $1.3 \%, p<0.0001$; respectively). The intra-abdominal injury rate was higher in the multi-regional stab wound group of patients $(40 \%$ vs. $35 \%, p=0.0004$ ) (Table 2).

Mortality was significantly higher $(2.5 \%$ vs. $0.9 \%, p=$ 0.0001 ) and mean LOS was longer in patients with multi-regional stab wounds $(5.2 \pm 6.5$ vs. $3.9 \pm 5.4$ days, $p<0.0001)$. Frequency of injury to the liver, kidney and spleen was higher in the multi-regional stab wound group $(13.2 \%$ vs. $8.4 \%, p<0.0001 ; 5.6 \%$ vs. $3.6 \%, p=$ $0.004 ; 8.2 \%$ vs. $1.2 \%, p<0.0001$; respectively). The small bowel was injured more frequently in the isolated abdominal stab wound group $(10.4 \%$ vs. $5.4 \%, p<0.001)$ (Table 3).

Independent adjusted risk factors for intra-abdominal injury were systolic blood pressure $\leq 90 \mathrm{mmHg}(\mathrm{OR}=$ $1.5(1.1-2.1, p=0.02), \mathrm{GCS}<13(\mathrm{OR}=3.9(2.4-6.2, \mathrm{p}<$ $0.001)$, age $\geq 50$ years $(\mathrm{OR}=2.2(1.01-4.6, p=0.048)$ and multi-regional stab wounds $(\mathrm{OR}=1.3 \quad(1.1-1.6, \quad p<$ 0.001) (Table 4).

\section{Key results}

Patients with multi-regional stab wounds have a higher rate of intra-abdominal injuries compared with patients with isolated abdominal stab wounds.

\section{Discussion}

Until the late twentieth century, operative management was the only accepted standard for penetrating wounds to the abdomen. In 1969, in a study of 600 stabbed patients, Nance et al. [6] reported the advantage of a selective non operative approach compared to mandatory explorative laparotomy. Later, several studies supported evidence that not all penetrating abdominal wounds require surgery. Navsaria et al. [7] in a study on 186 stabbed trauma victims successfully managed $53.8 \%$ of 
Table 1 Baseline Characteristics, Injury Pattern and Outcomes ${ }^{a}$

\begin{tabular}{|c|c|c|c|c|}
\hline & \multicolumn{3}{|l|}{ n/n (\%) } & \multirow[t]{2}{*}{$p$ Value } \\
\hline & Isolated ASW & Multiregional SW & All & \\
\hline \multicolumn{5}{|l|}{ Demographics } \\
\hline Age $\geq 50$ & $88 / 1866$ (4.7\%) & $118 / 2088(5.7 \%)$ & 206/3954 (5.2\%) & 0.19 \\
\hline Male gender & 1786/1870 (95\%) & 1993/2093 (95\%) & $3779 / 3963$ (95\%) & 0.67 \\
\hline \multicolumn{5}{|l|}{ Injury characteristic } \\
\hline $\mathrm{SBP}<90 \mathrm{mmHg}$ & 101/1852 (5.5\%) & 193/2072 (9,3\%) & 294/3924 (7.5\%) & $<0.0001$ \\
\hline $\mathrm{GCS} \leq 8$ & 24/1833 (1.3\%) & $83 / 2046(4.1 \%)$ & 107/3879 (2.8\%) & $<0.0001$ \\
\hline Abdomen AIS $\geq 3$ & $443 / 1871$ (24\%) & 517/2093 (25\%) & 960/3964 (24\%) & 0.45 \\
\hline Intra-abdominal injury & $660 / 1871$ (35\%) & $852 / 2093(40 \%)$ & 1512/3964 (38\%) & 0.0004 \\
\hline$I S S \geq 25$ & 19/1871 (1\%) & 197/2093 (9.4\%) & $216 / 3964$ & $<0.0001$ \\
\hline \multicolumn{5}{|l|}{ Outcomes } \\
\hline Mortality & 17/1871 (0.9\%) & $53 / 2093(2.5 \%)$ & 70/3964 (1.8\%) & 0.0001 \\
\hline LOS, mean (SD) & $3.9 \pm 5.4(n=1866)$ & $5.2 \pm 6.5(n=2077)$ & $4.6 \pm 6.0(n=3943)$ & $<0.0001$ \\
\hline
\end{tabular}

them non-operatively. Friedmann [8] in a study of 108 victims with abdominal SW found that a policy of mandatory laparotomy would have resulted in a negative laparotomy rate of $70 \%$. Renz and Feliciano [9] in a prospective observational study found a complication rate of $42 \%$ in patients who underwent non-therapeutic laparotomy for trauma. Later, a selective non operative approach was extended not only to anterior abdominal wall wounds, but also to flank and back injuries. Ocampo et al. [10] in a study of 473 patients stabbed to the posterior abdomen demonstrated a $76 \%$ success rate with conservative management. Increased liberal use of CT in such cases demonstrated a high accuracy for the presence of intra-abdominal injury and even peritoneal violation $[11,12]$. In order to avoid missing serious, but initially asymptomatic injuries, several diagnostic algorithms have been suggested. These algorithms take into consideration physical examination, local wound exploration, diagnostic peritoneal lavage and/or imaging. Eastern Association for the Surgery of Trauma guidelines strongly recommend considering abdominal $\mathrm{CT}$ in patients with abdominal stab wounds that have been selected for conservative management [5]. Nevertheless, potential risk of exposure to ionized radiation causing by CT should not be ignored [11, 13]. Inaba et al. [14] prospectively compared CT against serial physical examination in the evaluation of 249 patients with a stab wound to the abdomen. In their study, CT findings did not alter clinical decision making. The sensitivity and specificity of physical examination were 100.0 and $98.7 \%$, respectively, while those of CT were 31.3 and $84.2 \%$, respectively. The authors concluded that a physical examination-based diagnostic algorithm is effective and decreased the radiation burden in the management of patients with abdominal stab wounds.

Little is known about the therapeutic approach in the management of patients with multiple stab wounds in different body areas including the abdomen compared with isolated abdominal wounds. We live in a world where crime and the severity of violence consistently worsen over the years. Kessel et al. [15] in a study based on the national trauma database showed a gradual increase in both the absolute rate and the relative incidence of serious stab injuries in Israel. The authors also found that $50 \%$ of the victims suffered from multiple stab wounds to different body areas. A similar tendency has been reported in other countries $[16,17]$.

In the current study, we assumed that the frequency of intra-abdominal organ injury in patients with multi-regional stab wounds may be higher than that reported in the

Table 2 Comparison of Penetration/Penetration with Intra-abdominal Injury Incidence in Patients with Isolated Versus Multiple Anatomical Area Stab Wounds

\begin{tabular}{|c|c|c|c|c|}
\hline Type of injury & Abdominal and other area $(n=2093)$ & Abdominal only $(n=1871)$ & Total $(n=3964)$ & $p$ Value \\
\hline No penetration & $693(33.1 \%)$ & $568(30.5 \%)$ & 1261 & 0.063 \\
\hline Penetration only & $548(26.2 \%)$ & $643(34.4 \%)$ & 1191 & $<0.001$ \\
\hline Penetration with intra-abdominal injury & $852(40.7 \%)$ & $660(35.3 \%)$ & 1512 & 0.0004 \\
\hline
\end{tabular}


Table 3 Comparison of Intra-abdominal Injury Abdominal Stab Wound Patients with or without Other Area Involvement

\begin{tabular}{llll}
\hline Organ & Abdominal and other area $(n=2093)$ & Abdominal only $(n=1871)$ & $p$ Value \\
\hline Vascular & $73(3.5 \%)$ & $80(4.3 \%)$ & 0.2 \\
Liver & $276(13.2 \%)$ & $158(8.4 \%)$ & $<0.0001$ \\
Kidney & $117(5.6 \%)$ & $68(3.6 \%)$ & 0.004 \\
Spleen & $171(8.2 \%)$ & $36(1.9 \%)$ & $<0.001$ \\
Stomach & $92(4.4 \%)$ & $77(4.1 \%)$ & 0.66 \\
Pancreas & $16(0.8 \%)$ & $24(1.3 \%)$ & 0.103 \\
Small bowel & $213(10.1 \%)$ & $516(27.5 \%)$ & $<0.001$ \\
Colon/rectum & $97(4.6 \%)$ & $109(5.8 \%)$ & 0.09 \\
\hline
\end{tabular}

literature for patients with isolated abdominal stab wounds. We hypothesized that multiple stabs reflected increased aggression by the attacker, that would resulted in increased severity of the injuries. About $30 \%$ of our study population had no penetration into the abdominal cavity. Two thirds of all stab wounds patients in our study had violation of the abdominal cavity with or without intra-abdominal organ injuries. Similar findings have been reported in other studies [18, 19]. However, the major finding of this work was a significantly higher incidence of most intra-abdominal organ injuries in patients with multiregional stab wounds.

We were unable to find similar studies in the relevant English medical literature According to the literature, the most commonly injured organs in patients with stab wounds are the liver and small bowel. Each of them comprises $30 \%$ of all intra-abdominal injuries [20]. In our study, the overall incidence of solid organ injury was lower, but frequency these organs injury was significantly higher in patients with multiple body areas stab wounds.

Clinical observation and serial abdominal examination is a worldwide accepted approach to the patient with isolated abdominal SW [14]. Nevertheless it may not be appropriate in patients with multiple stab wounds due to relatively high probability of missing intra-abdominal injury.

This tendency may have an impact on current acceptable management approach to the patient with abdominal stab wound. Presence of additional to abdomen stab wounds may complicate the patient manage because of various aspects. Other wounds, even superficial, may be the source of significant pain that often requires more pain medications, sedation or in some cases even intubation. Major wounds may draw the physician attention and seem to be of major priority, thereby delay treatment of life-threatening injuries. Moreover, such injuries make the clinical observation and serial examination very limited or even impossible in some cases.

\section{Study limitations}

The trauma registry does not include patients discharged from emergency departments. Therefore, the real incidence of non-penetrating abdominal stab wounds is unknown. However, in these mildly injured patients, the presence of other anatomical areas wounds has no impact on the abdominal management protocol.

The second limitation of this study is that we had no complete information regarding injuries aside from the abdomen. Nevertheless, we assume that the variability between the groups should be minimized by the large sample size provided by the registry.

\section{Conclusions}

The incidence of almost all intra-abdominal organ injuries in patients with multi-regional stab wounds was significantly higher compared to isolated abdominal stab wounds. These findings may support the development of different diagnostic approaches to patients with multiple body areas stab wounds. Based on the study results we conclude that only clinical observation may not be an appropriate management approach in patients with multi-regional stab wounds. We believe that abdominal

Table 4 Univariate versus Multivariate Analysis of Risk Factors for Intra-abdominal Injury in Patients with Abdominal Stab Wounds

\begin{tabular}{lllll}
\hline Variable & Unadjusted & $p$-Value & Adjusted $^{\text {a }}$ & $p$ Value \\
\hline SBP $\leq 90$ mm HG & $2.24(1.7-2.9)$ & $<0.001$ & $1.5(1.1-2.1)$ & 0.02 \\
GCS $<13$ & $6.2(4.2-9.3)$ & $<0.001$ & $3.9(2.4-6.2)$ & $<0.001$ \\
Age $\geq 50$ & $3.3(1.7-6.1)$ & $<0.001$ & $2.2(1.01-4.6)$ & 0.048 \\
Female gender & $1.24(0.9-1.7)$ & 0.16 & $1.16(0.8-1.7)$ & 0.45 \\
Multiregional stab wounds & $1.24(1.1-1.4)$ & 0.001 & $1.3(1.1-1.6)$ & $<0.001$ \\
\hline
\end{tabular}

SBP systolic blood pressure, GCS Glasgow Coma Scale

${ }^{a}$ Regression odds ratio compared with: systolic blood pressure $90 \mathrm{mmHg}$ and above, GCS 15, age group 0-14 years, male gender, abdominal-only stab wounds 
CT in patients with abdominal and other anatomical body areas stab wounds should be considered. Future prospective studies will assist to develop unique management protocol for these patients.

\section{Abbreviations}

AIS: Abbreviated Injury Score; CT: Computerized Tomography; GCS: Glasgow Comma Scale; ISS: Injury Severity Score; LOS: length of stay; SBP: Systolic Blood Pressure; SW: Stab Wound

\section{Acknowledgements}

The authors would like to thank the members of Israel Trauma Group for their help in data accumulation.

The Israeli Trauma Group includes

Hany Bahouth, MD

Alexander Becker, MD

Ben Ely Michael, MD

Alexander Braslavsky, MD

Igor Jeroukhimov, MD

Milad Qarawany, MD

Boris Kessel, MD

Yoram Klein, MD

Guy Lin, MD

Ofer Merin, MD

Miklosh Bala, MD

Youri Mnouskin, MD, PhD

Avraham I. Rivkind, MD

Gad Shaked, MD

Dror Soffer, MD

Michael Stein, MD

Michael Weiss, MD.

Affiliations of The Israeli Trauma Group: Trauma and Emergency Surgery Division of General Surgery, Rambam Health Care Campus, Haifa, Israel (Bahouth); Department of Surgery, HaEmek Medical Center, Afula, Ruth and Bruce Rappaport Medical School, Technion, Haifa, Israel (Becker); Wolfson Medical Center, Holon, Israel (Ben Ely); Trauma Unit, Ziv Medical Center, Zfat, Israel (Braslavsky); Assaf Harofeh Medical Center, Tel Aviv University, Tel Aviv, Israel (Jeroukhimov); Trauma Unit, Poriya Medical Center, Tiberias, Israel (Qarawany); Trauma Unit, Hillel Yaffe Medical Center, Hadera, Israel (Kessel); Bruce Rappaport Medical School, Technion, Haifa, Israel, Divison of Acute Care Surgery and Trauma, Chaim Sheba Medical Center, Tel Hashomer, Israel (Klein); Kaplan Medical Center, Rehovot, Israel (Lin); Shaare Zedek Medical Center, Jerusalem, Israel (Merin); Hadassah Medical Center, Jerusalem, Israel (Bala, Rivkind); Surgery Department, Barzilai Medical Center, Ashkelon, Israel (Mnouskin); Trauma Unit, Soroka Medical Center, Beer-Sheva, Israel (Shaked); The Yitzhak Rabin Trauma Division, Division of Surgery, Tel Aviv Sourasky Medical Center, Tel Aviv, Israel (Soffer); Trauma Unit, Rabin Medical CenterBeilinson Hospital, Petah Tikva, Israel (Stein): Trauma Unit, Galilee Medical Center, Nahariya, Israel (Weiss)

\section{Availability of data and materials}

Data is property of authors and could be available by request at igorjer65@gmail.com.

\section{Authors' contributions}

IJ and IW contributed equally to this work. IJ, YH and BK designed the research. YH, KP and AG performed the research and analyzed the data. ZS and RA collected data and performed statistical analysis. IJ and IW wrote the paper. All authors made important intellectual contributions to the manuscript, and all authors approved the final version before submission.

\section{Ethics approval and consent to participate}

This study was based on existing data from Israeli National Trauma Registry. This institute data collection performed without identifiers for the purposes of quality assessment and research. Studies done based on these data are waived from institutional review board approval.

\section{Competing interests}

The authors declare that they have no competing interests.

\section{Publisher's Note}

Springer Nature remains neutral with regard to jurisdictional claims in published maps and institutional affiliations.

\section{Author details}

${ }^{1}$ Trauma Unit, Assaf Harofeh Medical Center, Zerifin 7030000, Israel, affiliated with the Sackler Faculty of Medicine, Tel Aviv University, Tel Aviv, Israel. ${ }^{2}$ National Center for Trauma and Emergency Medicine Research, Gertner Institute for Epidemiology and Health Policy Research, Sheba Medical Center, 52621 Tel Hashomer, Israel. ${ }^{3}$ Surgical Division, Hillel Yaffe Medical Center, 38100 Hadera, Israel. ${ }^{4}$ Trauma Unit, Hillel Yaffe Medical Center, 38100 Hadera, Israel.

Received: 13 February 2018 Accepted: 1 June 2018

Published online: 27 June 2018

\section{References}

1. Pallett JR, Sutherland E, Glucksman E, Tunnicliff M, Keep JW. A crosssectional study of knife injuries at a London major trauma Centre. Ann R Coll Surg Engl. 2014;96:23-6.

2. Biffl WL, Kaups KL, Pham TN, et al. Validating the western trauma association algorithm for managing patients with anterior abdominal stab wounds: a western trauma association multicenter trial. J Trauma. 2011;71:1494.

3. Benjamin E, Demetriades D. Nonoperative Management of Penetrating Injuries to the abdomen. Curr Trauma Rep. 2015;1(2):102-6.

4. Biffl WL, Leppaniemi A. Management guidelines for penetrating abdominal trauma. World J Surg. 2015;39(6):1373-80.

5. Como JJ, Bokhari F, Chiu WC, Duane TM, Holevar MR, Tandoh MA, et al. Practice management guidelines for selective nonoperative management of penetrating abdominal trauma. J Trauma. 2010;68:721-33.

6. Nance FC, Cohn I. Surgical judgment in the management of stab wounds of the abdomen. Ann Surg. 1969;170:569-80.

7. Navsaria PH, Berli JU, Edu S, Nicol AJ. Non-operative management of abdominal stab wounds-an analysis of 186 patients. S Afr J Surg. 2007:45:128-30. 32

8. Friedmann P. Selective management of stab wounds of the abdomen. Arch Sur. 1968;96:292-5.

9. Renz BM, Feliciano DV. The length of hospital stay after an unnecessary laparotomy for trauma: a prospective study. J Trauma. 1996;40:187-90.

10. Ocampo H, Yamaguchi M, Mackabee J, Ordog G, Fleming A. Selective management of posterior stab wounds. J Natl Med Assoc. 1987;79:283-8.

11. Salim A, Sangthong B, Martin M, Brown C, Plurad D, Inaba K, et al. Use of computed tomography in anterior abdominal stab wounds. Arch Surg. 2006;141:745-50. discussion 750-2

12. Fletcher TB, Setiawan H, Harrell RS, Redman HC. Posterior abdominal stab wounds: role of CT evaluation. Radiology. 1989;173:621-5.

13. Holmes JF, McGahan JP, Wisner DH. Rate of intra-abdominal injury after a normal computed tomographic scan in adults with blunt trauma. Am J Emerg Med. 2012:3:574-9.

14. Inaba K, Okoye OT, Rosenheck R, Melo N, Branco BC, Talving P, et al. Prospective evaluation of the role of computed tomography in the assessment of abdominal stab wounds. JAMA Surg. 2013;148:810-6.

15. Kessel B, Peleg K, Hershekovitz Y, Khashan T, Givon A, Ashkenazi I, et al. Abdominal stab wounds in Israel, 1997-2004: significant increase in overall incidence and prevalence of severe injury. Isr Med Assoc J. 2008;10:135-7.

16. Casali M, Di Saverio S, Tugnoli G, Biscardi A, Villani S, Cancellieri F, et al. [Penetrating abdominal trauma: 20 years experience in a western European trauma center]. [article in Italian]. Ann Ital Chir. 2008:79:399-407.

17. Rozen WM, Ma EH, Jones IT, Judson RT. Emerging epidemic in Australia: abdominal stab wounds. Twenty-four months at a major trauma Centre. Emerg Med Australas. 2007;19:262-8.

18. Cothren CC, Moore EE, Warren FA, Kashuk JL, Biffl WL, Johnson JL. Local wound exploration remains a valuable triage tool for the evaluation of anterior abdominal stab wounds. Am J Surg. 2009;198:223-6.

19. Omari A, Bani-Yaseen M, Khammash M, Qasaimeh G, Eqab F, Jaddou H. Patterns of anterior abdominal stab wounds and their management at Princess Basma teaching hospital, north of Jordan. World J Surg. 2013:37:1162-8.

20. Chestovich PJ, Browder TD, Morrissey SL, Fraser DR, Ingalls NK, Fildes JJ. Minimally invasive is maximally effective: diagnostic and therapeutic laparoscopy for penetrating abdominal injuries. J Trauma Acute Care Surg. 2015:78:1076-83. discussion 1083-5 